\title{
CORRECTIONS TO THE PAPER "REMARKS ON FLUCTUATIONS OF SUMS OF INDEPENDENT RANDOM VARIABLES"
}

K. L. CHUNG AND M. KAC

Dr. Erdös has called to our attention that the results (1.4) and (1.5) of our paper cited above (Memoirs of the American Mathematical Society, no. 6) are incorrect. The correct results are as follows:

If $\alpha<1$,

$$
\frac{N_{n}}{\log n} \rightarrow 2 D(\alpha) \text { in probability }
$$

and if $\alpha=1$,

$$
\frac{N_{n}}{(\log n)^{2}} \rightarrow 2 \pi^{2} \text { in probability. }
$$

We shall prove only (1) here. Consider, as in the paper, $\bar{N}_{n}$ which is the number of $s_{k}$ 's, $1 \leqq k \leqq n$, for which $s_{k}>0$ and $s_{k+1}<0$. The calcution given on pp. 7-8 of our paper is correct for $c=0$ and gives

$$
E\left\{\bar{N}_{n}\right\} \sim D(\alpha) \log n
$$

where

$$
D(\alpha)=\frac{\Gamma(\alpha+1) \sin (\pi \alpha / 2)}{\pi \alpha} \int_{0}^{\infty} \frac{\rho(x)}{x^{\alpha}} d x .
$$

For $c \neq 0$ the calculation is invalid and now we shall proceed to give the correct evaluation of the second moment.

We have

$$
\begin{aligned}
& E\left\{\bar{N}_{n}^{2}\right\}=E\left\{\bar{N}_{n}\right\} \\
& +2 \sum_{1 \leq k<k+1<l \varliminf_{n-1}} \operatorname{Pr}\left\{s_{k}>0, s_{k+1}<0, s_{l}>0, s_{l+1}<0\right\}, \\
& \operatorname{Pr}\left\{s_{k}>0, s_{k+1}<0, s_{l}>0, s_{l+1}<0\right\} \\
& =\iint_{0}^{\infty} \iint \rho_{k}(u) \rho(u+v) \rho_{l-k-1}(v+x) \rho(x+y) d x d y d u d v .
\end{aligned}
$$

By a simple change of variables we find that the sum in (5) can be written as

Received by the editors November 21, 1952. 


$$
\begin{aligned}
\sum_{k=1}^{n-2} k^{1 / \alpha} & \int_{0}^{\infty} \int_{0}^{\infty} \rho(u) \rho\left(k^{1 / \alpha}(u+v)\right) d u d v \\
& \sum_{j=1}^{n-k-1} j^{1 / \alpha} \int_{0}^{\infty} \int_{0}^{\infty} \rho\left(\left(\frac{k}{j}\right)^{1 / \alpha} v+x\right) \rho\left(j^{1 / \alpha}(x+y)\right) d x d y .
\end{aligned}
$$

For a sufficiently small $\epsilon>0$ we split the second sum into three parts:

$$
\sum_{j=1}^{n-k-1}=\sum_{j=1}^{[\epsilon k]}+\sum_{j=[\epsilon k]+1}^{[k / \epsilon]}+\sum_{j=[k / \epsilon]+1}^{n-k-1} .
$$

In the estimates below we shall repeatedly use two properties of $\rho(x)$. (A) For all $x \neq 0$

$$
\rho(x)<\frac{A}{x^{1+\alpha}}
$$

where $A$ is an absolute constant. This follows from Polya's formula. (B) For all $x>0$ and $x^{\prime}>0$

$$
\rho\left(x+x^{\prime}\right) \leqq \rho(x) .
$$

This follows from the unimodality of $\rho(x)$, proved by Wintner [1].

We begin with the second sum in (7). Using (A) and (B) we see that it is less than

$$
A \sum_{\epsilon<j \leqq k / \epsilon} \frac{1}{j} \int_{0}^{\infty} \int_{0}^{\infty} \frac{\rho(x)}{(x+y)^{1+\alpha}} d x d y \leqq A \log \frac{1}{\epsilon},
$$

where as later we shall use $A$ to denote a changeable constant possibly depending on $\alpha$. Hence the contribution of this part to (6) is less than

$$
A \log \frac{1}{\epsilon} \sum_{k=1}^{n-2} \frac{1}{k} \int_{0}^{\infty} \int_{0}^{\infty} \frac{\rho(u)}{(u+v)^{1+\alpha}} d u d v \leqq A \log \frac{1}{\epsilon} \log n .
$$

Next, the first sum in (7) is less than

$$
A \sum_{j=1}^{[\epsilon]} \frac{1}{j} \int_{0}^{\infty} \int_{0}^{\infty} \rho\left(\left(\frac{1}{\epsilon}\right)^{1 / \alpha} v+x\right) \frac{d x d y}{(x+y)^{1+\alpha}} .
$$

By (B) we have if $v \geqq \epsilon$

$$
\rho\left(\left(\frac{1}{\epsilon}\right)^{1 / \alpha} v+x\right) \leqq \rho(G+x)
$$

where $G=\epsilon^{1-1 / \alpha}$. The contribution of (9) for this range of $v$ to (6) is therefore less than 


$$
A \sum_{k=1}^{n-2} \frac{\log k}{k} \int_{0}^{\infty} \frac{\rho(G+x)}{x^{\alpha}} d x \leqq A \log ^{2} n \int_{0}^{\infty} \frac{\rho(G+x)}{x^{\alpha}} d x .
$$

Since $G \rightarrow \infty$ as $\epsilon \rightarrow 0$, by Lebesgue's dominated convergence theorem this is less than

$$
\delta_{1}(\epsilon) \log ^{2} n
$$

where $\delta_{1}(\epsilon) \rightarrow 0$ as $\epsilon \rightarrow 0$.

For $v \leqq \epsilon$ we have

$$
\rho\left(\left(\frac{1}{\epsilon}\right)^{1 / \alpha} v+x\right) \leqq \rho(x),
$$

hence (9) is less than $A \log k$ and the contribution to (6) is less than $A \sum_{k=1}^{n-2} \frac{\log k}{k} \int_{0}^{\infty} \rho(u) d u \int_{0}^{\epsilon} \frac{d v}{(u+v)^{1+\alpha}}$

$$
\leqq A \log ^{2} n\left[\int_{0}^{\infty} \frac{\rho(u)}{u^{\alpha}} d u-\int_{0}^{\infty} \frac{\rho(u)}{(u+\epsilon)^{\alpha}} d u\right] .
$$

Putting $u+\epsilon=u^{\prime}$ in the last integral and using (B) again, we see that this is less than

$$
\begin{aligned}
A \log ^{2} n\left[\int_{0}^{\infty} \frac{\rho(u)}{u^{\alpha}} d u-\int_{\epsilon}^{\infty} \frac{\rho(u)}{u^{\alpha}} d u\right] \\
=A \log ^{2} n \int_{0}^{e} \frac{\rho(u)}{u^{\alpha}} d u=\delta_{2}(\epsilon) \log ^{2} n
\end{aligned}
$$

where $\delta_{2}(\epsilon) \rightarrow 0$ as $\epsilon \rightarrow 0$.

Finally, we consider the third sum in (7) which will yield the principal term. For $v>\epsilon^{-1}$ this sum is less than

$$
\begin{aligned}
A \sum_{j=[k / \epsilon]+1}^{n-k-1} \frac{1}{j} \int_{0}^{\infty} \int_{0}^{\infty} \rho(x) \frac{d x d y}{(x+y)^{1+\alpha}} & \\
& \leqq A \sum_{j=[k / \epsilon]+1}^{n-k-1} \frac{1}{j} \leqq A \log \frac{n-k}{k} .
\end{aligned}
$$

Hence the contribution to (6) is less than

$$
\begin{aligned}
A \sum_{k=1}^{n-2} \frac{1}{k} \log \frac{n-k}{k} \int_{0}^{\infty} \rho(u) d u \int_{\epsilon^{-1}}^{\infty} & \frac{d v}{(u+v)^{1+\alpha}} \\
& \leqq A \epsilon^{\alpha} \log ^{2} n=\delta_{3}(\epsilon) \log ^{2} n
\end{aligned}
$$

where $\delta_{3}(\epsilon) \rightarrow 0$ as $\epsilon \rightarrow 0$. 
For $v \leqq \epsilon^{-1}$ we have by (B)

$$
\rho\left(\epsilon^{\prime}+x\right) \leqq \rho\left(\epsilon^{1 / \alpha} \nu+x\right) \leqq \rho(x)
$$

where $\epsilon^{\prime}=\epsilon^{(1 / \alpha)-1}$. Hence the contribution to (6) as $n \rightarrow \infty$ is asymptotically less than

$$
C^{2}(\alpha) \sum_{k=1}^{n-2} \frac{1}{k} \int_{0}^{\infty} \frac{\rho(u)}{u^{\alpha}} d u \sum_{j=[k / \epsilon]+1}^{n-k-1} \frac{1}{j} \int_{0}^{\infty} \frac{\rho(x)}{x^{\alpha}} d x
$$

and greater than

$$
C^{2}(\alpha) \sum_{k=1}^{n-2} \frac{1}{k} \int_{0}^{\infty} \frac{\rho(u)}{u^{\alpha}} d u \sum_{j=\{k / \epsilon]+1}^{n-k-1} \frac{1}{j} \int_{0}^{\infty} \frac{\rho\left(\epsilon^{\prime}+x\right)}{x^{\alpha}} d x
$$

where $C(\alpha)=(\pi \alpha)^{-1} \Gamma(\alpha+1) \sin (\alpha \pi / 2)$. By the dominated convergence theorem (using (B) again),

$$
\lim _{\alpha \rightarrow 0} \int_{0}^{\infty} \frac{\rho\left(\epsilon^{\prime}+x\right)}{x^{\alpha}} d x=\int_{0}^{\infty} \frac{\rho(x)}{x^{\alpha}} d x .
$$

It follows that this contribution is asymptotically

$$
\begin{aligned}
{\left[1-\delta_{4}(\epsilon)\right] D^{2}(\alpha) } & \sum_{k=1}^{n} \frac{1}{k}\left[\log (n-k)-\log \frac{k}{\epsilon}\right] \\
& =\left[1-\delta_{4}(\epsilon)\right] D^{2}(\alpha)\left[\frac{1}{2} \log ^{2} n+\log \epsilon \log n\right\rfloor+O(\log n)
\end{aligned}
$$

where $\delta_{4}(\epsilon) \rightarrow 0$ as $\epsilon \rightarrow 0$. Combining this with (8), (10), (11), and (12) we conclude that (6) is equal to

$$
\frac{1}{2} D^{2}(\alpha) \log ^{2} n+\delta(\epsilon) \log ^{2} n+O(\log \epsilon \log n)
$$

where $\delta(\epsilon) \rightarrow 0$ as $\epsilon \rightarrow 0$. Since $\epsilon$ is arbitrary this means that (6) is asymptotically equal to $(1 / 2) D^{2}(\alpha) \log ^{2} n$. It then follows from (3) and (5) that

$$
E\left\{\bar{N}_{n}^{2}\right\} \sim D^{2}(\alpha) \log ^{2} n
$$

From (3) and (13) it follows that

$$
\bar{N}_{n} / \log n \rightarrow D(\alpha) \text { in probability }
$$

which is equivalent to the assertion (1).

\section{RefERENCE}

1. A. Wintner, Asymptotic distributions and infinite convolutions, Edwards Brothers, 1938, p. 32.

CORNELL UNIVERSITY 